Preface

\title{
Editorial Compilation III
}

\author{
Emmanuel J. Favaloro, PhD, FFSc (RCPA) ${ }^{1}$ Giuseppe Lippi, MD ${ }^{2}$ \\ 1 Department of Haematology, Institute of Clinical Pathology and \\ Medical Research (ICPMR), Westmead Hospital, Westmead, Australia \\ ${ }^{2}$ Section of Clinical Biochemistry, University of Verona, Verona, Italy
}

Semin Thromb Hemost 2017;43:4-7.

Welcome to another issue of Seminars in Thrombosis and Hemostasis. This is the third issue published under the "banner" of "Editorial Compilation." Although Seminars in Thrombosis and Hemostasis is primarily a theme-driven publication, the opportunity intermittently arises to publish issues containing more wide-ranging articles of current interest and controversy that do not quite match a current themed issue in progress. We also require a medium to enable publication of contributions from our Eberhard F. Mammen Young Investigator Award winners. ${ }^{1-5}$ The current issue has a mixture of content that comprises all these elements as well as broadly fitting within the separate themes of "thrombosis" and "bleeding."

This issue begins with the contributions from the two of our recent (2015) Eberhard F. Mammen Young Investigator Award winners. ${ }^{5}$ The first, from Dr Juraj Sokol and colleagues, ${ }^{6}$ is on the topic of "Sticky Platelet Syndrome," a particular favorite for this journal, considering it was a topic of major interest to the founding editor of the journal, Eberhard F. Mammen. The current authors initially discuss the etiology of thrombosis from the perspective of Virchow triad, proposing concerted roles for abnormalities in blood composition, vessel wall components, and blood flow in the development of arterial and venous thrombosis. The authors also note that recent work has been primarily focused on describing abnormalities in blood composition, including defects of coagulation proteins and platelets, and that prothrombotic platelet disorders are still less understood, despite defects of coagulation factors being relatively well described in the literature. One such defect is the sticky platelet syndrome, which the authors review, and furthermore propose a new definition and standardization of diagnostic criteria.

Continuing the theme of "thrombosis," another of the 2015 Young Investigators, Dr. Ljubica Jovanovic and her colleagues ${ }^{7}$ then explore the practical aspects of monitoring of antiplatelet therapy. Despite the application of newer antiplatelet drugs (prasugrel and ticagrelor), dual antiplatelet therapy with clopidogrel and aspirin remains the standard of care for patients with acute coronary syndrome (ACS) undergoing percutaneous coronary intervention $(\mathrm{PCI})$, especially in countries of lower socioeconomic status. Regardless of proven benefits, numerous studies have shown that certain groups of patients who receive standard doses of clopidogrel and aspirin do not respond adequately, and many of them also exhibit adverse cardiovascular events. Studies have also shown that the risk of stent thrombosis and ischemic complications is higher in patients with certain conditions, such as ACS, diabetes mellitus, thrombocytosis, reduced systolic function of the left ventricle, presence of multiple stents, longer and thinner stents, and renal failure. The authors contend that in such patients, it may be useful to assess their response to antiplatelet therapy, and perhaps to adjust such therapy based on their individual response. This provides an impetus for platelet function tests, which may also be furthermore useful to a second group of patients, those at increased risk of bleeding. These may include elderly patients, those with low body weight, anemia, thrombocytopenia, renal failure, past or current ventricular or duodenal ulcer, coagulopathy, or liver disease. A third potential application of platelet function tests in patients on antiplatelet therapy might be during preparation and evaluation of surgical interventions or invasive diagnostic procedures. In summary, the authors contend that for high-risk patients, the careful selection of optimal antiplatelet $\operatorname{drug}(\mathrm{s})$ and dose should be based on their personalized risk of thrombosis or bleeding, and that platelet function testing may play a role in such scenarios.

The next series of articles comprise either contributions from editorial members of Seminars in Thrombosis $\mathcal{E}$ Hemostasis or the best of recent unsolicited material. The first set of papers continues the "prothrombotic" theme. First, Boc and coworkers explore the impact of asymptomatic pulmonary embolism (PE) on the long-term prognosis of patients with deep venous thrombosis (DVT) and PE. ${ }^{8}$ The authors first remind readers that asymptomatic PE is present in at least one-third of patients presenting with DVT, but that knowledge about its influence on
Address for correspondence Emmanuel J. Favaloro, PhD, FFSc (RCPA), Department of

Haematology, Institute of Clinical Pathology and Medical Research (ICPMR), Westmead Hospital,

Westmead, Australia (e-mail: emmanuel.

favaloro@health.nsw.gov.au).
Issue Theme Editorial

Compilation III; Guest Editors: Emmanuel J. Favaloro, PhD, FFSc (RCPA), and Giuseppe Lippi, MD.
Copyright $\odot 2017$ by Thieme Medical Publishers, Inc., 333 Seventh Avenue, New York, NY 10001, USA. Tel: +1(212) 584-4662.
DOI http://dx.doi.org/ 10.1055/s-0036-1597649. ISSN 0094-6176. 
their prognosis is still limited. The aim of their study was to therefore assess the prognostic impact of asymptomatic PE in patients with DVT and to explore risk factors for recurrent venous thromboembolic events. A total number of 200 consecutive patients with the first episode of objectively confirmed DVT without symptoms of PE were included. All patients underwent ventilation-perfusion scintigraphy within 48 hours of DVT confirmation. Patients with inconclusive scans further underwent computed tomography (CT) pulmonary angiography. At the time of inclusion and 4 weeks after discontinuation of anticoagulation, the levels of biomarkers of hemostasis and inflammation were assessed. Patients were followed up for a mean period of $4.2 \pm 0.6$ years. Recurrent episodes of VTE were recorded. Consistent with the literature, asymptomatic PE was present in $33.5 \%$ of patients. During follow-up, 27 recurrent VTEs were recorded, 20 presenting as DVT and 7 presenting as symptomatic PE. Asymptomatic PE was not significantly associated with the rate of recurrence $(p=0.676)$, but recurrent events were associated with unprovoked versus provoked DVT (hazard ratio [HR]: 5.01; 95\% confidence interval $[\mathrm{CI}]: 2.25-11.17 ; p<0.001)$ and with increased versus normal D-dimer values, measured 4 weeks after discontinuation of anticoagulation (HR: 6.47; 95\% CI: 2.96-14.17; $p<0.001$ ).

Turcato and colleagues ${ }^{9}$ then present their recent findings around the use of red blood cell distribution width (RDW) as an independent predictor of clinical outcomes in patients with thrombotic disorders, in particular as potentially applied for appropriate and timely management, including early diagnosis and accurate prognostication of patients with acute ischemic stroke. The authors designed a retrospective observational study to investigate whether RDW might also retain predictive significance in stroke patients undergoing thrombolytic therapy. Based on all patients admitted to the Emergency Department (ED) of the University Hospital of Verona (Italy) with a diagnosis of ischemic stroke, who underwent systemic thrombolysis between January 2013 and June 2015, RDW along with basal clinical characteristics were recorded at ED admission. The final study population consisted of 328 patients. A significant association was found between stroke severity (National Institutes of Health Stroke Scale [NIHSS] score) and RDW $(r=0.322 ; p<0.001)$. The median RDW value in patients with clinical improvement after thrombolysis was significantly lower than in patients without (13.4 vs. $14.1 \% ; p<0.001$ ). The diagnostic accuracy (area under the curve) of RDW for predicting the lack of neurological improvement was 0.667. In univariate analysis, an RDW value $>14.5 \%$ was associated with increased rate of no neurological improvement (odds ratio [OR]: 2.38; 95\% CI: 1.37-4.13), an association remaining significant also in multivariate analysis (OR: 1.85; 95\% CI: 1.13-3.32). Survivor curve analysis showed that patients with RDW values $\geq 14.5 \%$ had a higher risk of 1 -year mortality and shorter survival. These results thus suggest that RDW assessment at ED admission may provide valuable diagnostic and prognostic information in patients with acute ischemic stroke, and provides additional clues about the potential interplay between anisocytosis and ischemic disorders.
Finally, in terms of potential prothrombotic risk, Boeddha and colleagues explore the concept of gene variations in the protein $\mathrm{C}$ and fibrinolytic pathways, and their relevance for severity and outcome in pediatric sepsis. ${ }^{10}$ The host response to infection involves complex interplays between inflammation, coagulation, and fibrinolysis. Deregulation of hemostasis and fibrinolysis is a major cause of critical illness and an important determinant of outcome in severe sepsis. The hemostatic response to infection varies widely between individuals, and is in part explained by polymorphisms in genes responsible for the protein $C$ and the fibrinolytic pathways. This review gives an overview of genetic polymorphisms in the protein $\mathrm{C}$ and fibrinolytic pathways associated with susceptibility and severity of pediatric sepsis. In addition, genetic polymorphisms associated with adult sepsis and other pediatric thromboembolic disorders are discussed, as these polymorphisms might be candidates for future molecular genetic research in pediatric sepsis.

This issue of Seminars in Thrombosis $\mathcal{E}$ Hemostasis then turns to topics around bleeding risk. First, Selle and coworkers $^{11}$ report a relatively large case series of platelet $\delta$ storage pool disease $(\delta-S P D)$, a platelet function disorder characterized by reduced number or content of dense granules. Reports on $\delta$-SPD are mostly limited to case presentations. The authors therefore aimed to retrospectively describe a series of 16 patients with congenital or acquired $\delta$-SPD to better characterize the disease. They assessed lumiaggregometry, $\alpha$ - and $\delta$-granules content, platelet ultrastructure, $\alpha_{\text {IIb }} \beta_{3}$ integrin, and glycoprotein Ib (GPIb) activation. Most patients generally demonstrate mild to moderate bleeding diathesis. Platelet aggregation studies showed moderate abnormalities with variable profiles, while all the individuals had almost complete absence of ATP release. Mepacrine capture, CD63 expression, and study of dense granules by electron microscopy enabled the authors to distinguish different subtypes of $\delta$-SPD with quantitative or qualitative defect. Surprisingly, significantly decreased GPIb expression levels after platelet activation with TRAP $50 \mu \mathrm{M}$ were found, suggesting that impaired mobilization of this glycoprotein may represent an additional feature of the disorder. The authors conclude that $\delta$-SPD represents a complex condition, characterized by multifaceted clinical and biological aspects, requiring a great deal of expertise to be properly diagnosed.

Carcao and colleagues then describe the development and use of the first recombinant factor XIII (rFXIII) for congenital factor XIII (FXIII) deficiency. ${ }^{12}$ Congenital FXIII deficiency is a rare, autosomal recessive bleeding disorder with potentially life-threatening consequences. FXIII is composed of two subunits (A and B), and a deficiency or dysfunction of either can result in protein deficiency. Traditionally, FXIII deficiency has been managed by infusing plasma-derived products containing FXIII (fresh frozen plasma, cryoprecipitate, and plasma-derived FXIII concentrates), all of which contain both subunits. Despite the increased safety of plasma-derived products, concern remains regarding potential viral safety issues. This review therefore describes the development, from concept to clinical use, of rFXIII (containing subunit A only; rFXIII- $A_{2}$ ) for congenital FXIII-A subunit deficiency. Unmet 
needs and ongoing challenges in congenital FXIII deficiency are also discussed. Despite the challenges in developing a product for a very rare bleeding disorder, the information gathered on efficacy, safety, and pharmacokinetics of FXIII replacement therapy represents the largest dataset on congenital FXIII-A subunit deficiency in the world. It also provides evidence for safety and efficacy of monthly prophylaxis with $35 \mathrm{IU} / \mathrm{kg}$ rFXIII-A $\mathrm{A}_{2}$ in patients with FXIII-A subunit deficiency. The issues encountered and overcome, along with lessons learned, may be applied to and encourage the development of new recombinant products for other rare bleeding disorders.

Franchini and colleagues then report on their recent findings that $\mathrm{O}$ blood group protects against inhibitor development in severe hemophilia A patients. ${ }^{13}$ The authors initially report that increasing evidence supports the link between $\mathrm{ABO}(\mathrm{H})$ blood group determinants and hemostasis. In particular, the $\mathrm{ABO}$-related different glycosylation pattern of von Willebrand factor (VWF) strongly influences its clearance and functional levels, and this may contribute to the interindividual variations in the half-life of infused factor VIII (FVIII) in hemophilia A (HA) patients. In the current study, the authors wished to investigate the role of $\mathrm{ABO}$ blood groups in regulating FVIII immunogenicity by evaluating their distribution in patients with severe (FVIII $<1 \mathrm{IU} / \mathrm{dL}$ ) HA according to inhibitor development and other known relevant factors. In a cohort of Italian severe HA patients $(n=209)$, the ABO blood group distribution was similar to that in the healthy general population. However, the distribution of inhibitors, developed in 56 patients overall (26.8\%), was significantly different for the four $A B O$ phenotypes $(0,18.2 \%$; $A, 31.9 \%$; $39.1 \%$; $A B, 25 \% ; p=0.033)$. This difference seemed more pronounced when only high-titer inhibitors (overall, $21.1 \%$ ) were considered (O, 11.4\%; A, 27.7\%; B, 34.8\%; $p=0.011$ ). Relative risks in 0 versus non-O blood group were 0.55 (95\% CI: $0.33-0.92)$ and 0.40 (95\% CI: 0.21-0.77) for any and hightiter inhibitors, respectively. In a multivariate logistic regression, O blood group was shown to lower ( $\sim 2$-fold) inhibitor risk, similarly with plasma-derived FVIII, whereas high-risk F8 mutations were associated with increased risk. However, the estimated effect of $\mathrm{O}$ blood type on inhibitor development was free from any significant correlation to other covariates, including presence of high-risk F8 mutations and type of replacement FVIII used. The authors conclude by summarizing that blood group $\mathrm{O}$ appears to protect against inhibitor development in their retrospective cohort of severe hemophiliacs, with independent effects from other covariates. However, they also recognize that larger prospective studies are needed to confirm this finding and to delve deeper into its pathophysiologic mechanisms.

The last two articles in the current issue of Seminars in Thrombosis \& Hemostasis deal with the most common inherited bleeding disorder, von Willebrand disease (VWD), caused by defect or deficiency of the plasma protein VWF. In the first of these studies, Sarah Just explores the past, current, and future state of play for VWF assays that measure platelet binding activity, with or without ristocetin. ${ }^{14}$ VWD was first described nearly a century ago in 1924 by Erik Adolf von
Willebrand. Diagnostic testing at the time was very limited. It was not until the mid to late 1900s that more tests become available to assist with the diagnosis and classification of VWD. Two of these tests are based on ristocetin, one being ristocetin-induced platelet aggregation (RIPA) and the other VWF ristocetin cofactor assay (VWF:RCo). The VWF:RCo provides functional assessment of in vitro VWF binding to the platelet glycoprotein (Gp) complex, GPIb-IX-V. Despite some advancements and newer technologies utilizing the principles of the original VWF:RCo assay, the original assay based on platelet agglutination is still referred to as the gold standard for measurement of VWF activity. This article reviews the history of VWD diagnostic assays, including RIPA and VWF:RCo over the past 40 years, as well as the newer assays that measure platelet binding with or without ristocetin, and which have been developed with the aim to potentially replace platelet-based ristocetin-dependent assays.

Continuing the theme of VWD, and suitably concluding this issue of the journal, Woods and colleagues describe their experience of phenotypic parameters in genotypically selected type 2B VWD (VWD2B) patients. ${ }^{15}$ VWD2B expresses gain-offunction mutations enhancing the binding of an individual's VWF to its platelet ligand, GPIb, and which are usually identified by increased RIPA. The authors describe the phenotypic profile of 38 genotypically selected VWD2B-affected family members (AFMs) belonging to 19 unrelated families. Major bleeding was observed in $68.4 \%$ of AFMs (previous to their diagnosis and registered by lifetime interviews), with a total of 46 episodes (1.21/patient), and was found to be highly related to the individual bleeding score and presence of thrombocytopenia, but otherwise unrelated to other laboratory parameters. Excessive mucocutaneous bleeding symptoms were often reported, the most frequent of which comprised menorrhagia, epistaxis, easy bruising, and bleeding after teeth extraction in oral cavity. Eight unaffected family members were also studied. The prevalence of VWD2B within families was 0.826 , and the penetrance of mutations was complete, making it mandatory to study entire family sets to complete diagnostic profiles. Seven heterozygous missense mutations were found, the most common being p.V1316M. In the p.R1308C group, $75 \%$ of the AFMs showed absent RIPA at $0.5 \mathrm{mg} / \mathrm{mL}, 66.6 \%$ of who had VWF:RCo $<10 \mathrm{IU} / \mathrm{dL}$, and $50 \%$ of who had VWF: $\mathrm{CB}<10 \mathrm{IU} / \mathrm{dL}$. In the $\mathrm{p} . \mathrm{S} 1310 \mathrm{~F}$ group, none of AFMs had VWF: $\mathrm{RCo} / \mathrm{Ag}<0.6$, but $100 \%$ had VWF: $\mathrm{CB} / \mathrm{Ag}<0.6$. Patients with either p.P1266L or p.R1304V were characterized as atypical VWD2B. Two de novo mutations were found in four AFMs belonging to two families. The authors also describe a previously unreported mutation p.Y1258C. Of their patients, 70.5\% had $\mathrm{O}$ blood group. In conclusion, the authors report that a normal VWF:RCo/Ag ratio and a negative RIPA at $0.5 \mathrm{mg} / \mathrm{mL}$ do not necessarily rule out a diagnosis of VWD2B.

As always, we would like to thank all the authors to this latest issue of "Editorial Compilations" for their original and comprehensive contributions. We also hope that you, representing the readership of this journal, find this issue of substantial interest. This will, of course, be determined in time, as measured and established for previous issues of this journal. 


\section{References}

1 Favaloro EJ. 2011 Eberhard F. Mammen award announcements. Semin Thromb Hemost 2011;37(5):431-439

2 Favaloro EJ. 2012 Eberhard F. Mammen award announcements. Semin Thromb Hemost 2012;38(5):425-432

3 Favaloro EJ. 2013 Eberhard F. Mammen award announcements. Semin Thromb Hemost 2013;39(6):567-574

4 Favaloro EJ. 2014 Eberhard F. Mammen award announcements: part II-Young Investigator Awards. Semin Thromb Hemost 2014; 40(7):718-723

5 Favaloro EJ. 2015 Eberhard F. Mammen Award announcements: part II-Young Investigator Awards. Semin Thromb Hemost 2015; 41(8):809-815

6 Sokol J, Skerenova M, Jedinakova Z, et al. Progress in the understanding of sticky platelet syndrome. Semin Thromb Hemost 2017;43(1):8-13

7 Jovanovic L, Antonijevic N, Novkovic T, et al. Practical aspects of monitoring of antiplatelet therapy. Semin Thromb Hemost 2017; 43(1):14-23

8 Boc A, Vene N, Košmelj K, Mavri A. Impact of asymptomatic pulmonary embolism on the long-term prognosis of patients with deep venous thrombosis. Semin Thromb Hemost 2017; 43(1):24-29

9 Turcato G, Cappellari M, Follador L, et al. Red blood cell distribution width is an independent predictor of outcome in patients under- going thrombolysis for ischemic stroke. Semin Thromb Hemost 2017;43(1):30-35

10 Boeddha NP, Emonts M, Cnossen MH, et al. Gene variations in the protein $C$ and fibrinolytic pathway: relevance for severity and outcome in paediatric sepsis. Semin Thromb Hemost 2017;43(1): 36-47

11 Selle F, James C, Tuffigo M, et al. Clinical and laboratory findings in patients with $\delta$-storage pool disease: a case series. Semin Thromb Hemost 2017;43(1):48-58

12 Carcao M, Fukutake K, Inbal A, et al. Developing the first recombinant factor XIII for congenital factor XIII deficiency: clinical challenges and successes. Semin Thromb Hemost 2017;43(1): 59-68

13 Franchini M, Coppola A, Mengoli C, et al; On Behalf of the Ad Hoc Study Group. Blood group O protects against inhibitor development in severe hemophilia A patients. Semin Thromb Hemost 2017;43(1):69-74

14 Just S. Laboratory testing for von Willebrand disease: the past, present, and future state of play for von Willebrand factor assays that measure platelet binding activity, with or without ristocetin. Semin Thromb Hemost 2017;43(1):75-91

15 Woods AI, Kempfer AC, Paiva J, et al. Phenotypic parameters in genotypically selected type $2 \mathrm{~B}$ von Willebrand Disease patients: a large single center experience including a new novel mutation. Semin Thromb Hemost 2017;43(1):92-100 